\title{
Effects of mixing eggs of different initial incubation time on the hatching pattern, chick embryonic development and post- hatch performance
}

\author{
Zhentao Zhong ${ }^{1}$, Yue Yu ${ }^{1}$, Shufang Jin ${ }^{1}$, Jinming Pan ${ }^{\text {Corresp. }}{ }^{1}$ \\ 1 Department of Biosystems Engineering, Zhejiang University, Hangzhou, China \\ Corresponding Author: Jinming Pan \\ Email address: panhouse@zju.edu.cn
}

Background. The hatch window that varies from 24 to $48 \mathrm{~h}$ is known to influence post-hatch performance of chicks. A narrow hatch window is needed for commercial poultry industry to acquire a high level of uniformity of chick quality. Hatching synchronization observed in avian species presents possibilities in altering hatch window in artificial incubation.

Methods. Layer eggs which were laid on the same day by a single breeder flock and stored for no more than two days started incubation 12 hours apart to obtain developmental distinction. The eggs of different initial incubation time were mixed as rows adjacent to rows on day 12 of incubation. During the hatching period (since day 18), hatching time of individual eggs and hatch window were obtained by video recordings. Embryonic development (day 18 and 20) and post-hatch performance up to day 7 were measured.

Results. The manipulation of mixing eggs of different initial incubation time shortened the hatch window of late incubated eggs in manipulated group by delaying the onset of hatching process, and improved the hatchability. Compared to the control groups, chick embryos or chicks in the egg redistribution group showed no significant difference in embryonic development and post-hatch performance up to day 7 .

Discussion. We have demonstrated that eggs that incubated with advanced eggs performed a narrow spread of hatch with higher hatchability, normal embryonic development as well as unaffected chick quality. This specific manipulation is applicable in the industrial poultry production to shorten hatch window and improve uniformity of chick quality. 
1 Effects of mixing eggs of different initial incubation time on the hatching pattern, chick

2

3 embryonic development and post-hatch performance

4

${ }^{1}$ Department of Biosystems Engineering and Food Science, Zhejiang University, Hangzhou,

$5 \quad 310058$, China

6

7 *Corresponding author: Dr. Jinming Pan, Department of Biosystems Engineering, Zhejiang

8 University, Hangzhou 310058, China.E-mail: panhouse@zju.edu.cn 


\section{ABSTRACT}

Background. The hatch window that varies from 24 to $48 \mathrm{~h}$ is known to influence post-hatch performance of chicks. A narrow hatch window is needed for the commercial poultry industry to acquire a high level of uniformity of chick quality. Hatching synchronization observed in avian species presents possibilities in altering the hatch window in artificial incubation.

Methods. Layer eggs which were laid on the same day by a single breeder flock and stored for no more than two days started incubation 12 hours apart to obtain developmental distinction. The eggs of different initial incubation time were mixed as rows adjacent to rows on day 12 of incubation. During the hatching period (since day 18), hatching time of individual eggs and hatch window were obtained by video recordings. Embryonic development (day 18 and 20) and posthatch performance up to day 7 were measured.

Results. The manipulation of mixing eggs of different initial incubation time shortened the hatch window of the late incubated eggs in the manipulated group by delaying the onset of the hatching process, and improved the hatchability. Compared to the control groups, chick embryos or chicks in the egg redistribution group showed no significant difference in embryonic development and post-hatch performance up to day 7 .

Discussion. We have demonstrated that eggs that incubated with advanced eggs performed a narrow spread of hatch with higher hatchability, normal embryonic development as well as unaffected chick quality. This specific manipulation is applicable in the industrial poultry production to shorten hatch window and improve uniformity of chick quality. 


\section{INTRODUCTION}

In artificial incubation, the inherent characteristics of eggs (e.g. parental age, egg weight and egg storage time) and incubation conditions (temperature and $\mathrm{CO}_{2}$ concentration during the hatching phase) play a crucial role in embryonic development that results in the spread of hatch (De Smit et al. 2006; Ipek \& Sozcu 2017; Maatjens et al. 2014; Nangsuay et al. 2016; Tona et al. 2003; Tona et al. 2007; Willemsen et al. 2010b). The spread of hatch is evaluated as the degree of hatching synchrony which essentially contributes to the uniformity of newly hatched chicks. In general, hatch window which defined as the time between early-hatching and late-hatching varies from 24 to 48 hours (Careghi et al. 2005; Decuypere et al. 2001). Thus, early hatched chicks will be held in the incubators with deprivation of feed and water until the entire batch of chicks hatch, rather than removed immediately upon hatching. The variability of delayed time with access to feed and water depressed the uniformity of post-hatch performance of the hatched chicks, including organ development, immune system activation, digestive enzyme stimulation and relative growth post hatch (Tona et al. 2003; Willemsen et al. 2010a).

In the nature, precocial avian species can achieve a narrow hatch window by acceleration (Holmberg 1991; Vince 1964) or retardation (Vince 1968) of hatching. This adaptive advantage of hatch synchrony enables the offsprings to avoid being abandoned by the parent bird and exposed to predators. Moreover, intraclutch hatch synchronization was found in the lesser snow goose (Davies \& Cooke 1983), pheasants and mallard ducks (Persson \& Andersson 1999), by shortening or prolonging the incubation period. In addition to this effect of sibling contact, the hatch process could also be affected by mixing eggs of different embryo developmental trajectory (Tona et al. 
50

2013). However, no effective manipulations during incubation have been performed to shorten hatch window in commercial poultry production.

Therefore, the aim of the present study was to achieve a narrowed hatch window through the manipulation of mixing eggs of different growth curves. In addition, potential effects on embryonic development and post hatch performance were studied. Hatching time of individual chicks, hatch window, hatchability, yolk residue and organ development, body weight and leg bone development were compared between the control and manipulated groups.

\section{MATERIALS AND METHODS}

All procedures in this study were approved by the committee of Care and Use of Animals of Zhejiang University, Hangzhou, China.

\section{Experimental design}

Hatching eggs ( $\mathrm{n}=680$; weight range from 53 to $57 \mathrm{~g}$ ) were obtained from a Hyline breeder flock at 35 to 36 weeks of age (Shenhai Breeding, Shenyang, China) and all eggs were laid on the same day and stored for no longer than 2 days. The eggs were marked with numbers and divided into early incubation group (EI) and late incubation group (LI). LI started incubation 12 hours later than EI so that the biological age (BA, calculated from the initial incubation time) of EI was $12 \mathrm{~h}$ older than that of LI. On BA $12 \mathrm{~d}$ of LI (BA $12.5 \mathrm{~d}$ of EI), 154 eggs randomly chosen from both EI and LI were distributed into the third incubator and defined as the manipulated incubation group (MI). The remaining eggs in EI and LI were regarded as control groups. The eggs in MI were distributed with EI rows adjacent to LI rows. On BA 18 of LI, the MI group was separated into EMI group (early incubated eggs in MI) and LMI group (late incubated eggs in MI) based on the 
71 previous marks. The separation was for distinguishing the origin groups of newly hatched chicks

72 in the hatching stage. The numbers of eggs in each group were 186 (EI), 186 (LI), 154 (EMI) and

73154 (LMI).

74

75

\section{Incubation}

The eggs were incubated and hatched in lab-scale incubators (NK-hatching, Dezhou Nongke Incubation Equipment Co. Ltd., Shandong, China) measuring $1100 \times 1000 \times 900 \mathrm{~mm}$ with a capacity of 352 eggs. The incubators were calibrated by a standard thermometer and hygrometer before egg incubation. The incubation was maintained at a temperature of $37.8 \pm 0.1{ }^{\circ} \mathrm{C}$ and a relative humidity around $60 \%$. The turning time interval during incubation was two hours until day 18 . Eggs were candled (Cool-Lite tester, GQF) on day 18 and those with a living embryo were transferred to hatching baskets. Fisheye cameras (DS-2CD3942F-I, HIKVISION) focused upon the hatching baskets were used to monitor the hatch process. All incubations stopped at BA $504 \mathrm{~h}$ of LI and the chicks were removed from the incubators.

\section{Post-hatch housing and management}

A total of 128 newly hatched chicks (32 per incubation group) were sampled and transferred to four pens of $1 \mathrm{~m}^{2}$ covered with sawdust. Artificial lightning was set for $23 \mathrm{~h} / \mathrm{d}$ from day 0 to 7 (40 lux at chick's eye level). Temperature was set to $34^{\circ} \mathrm{C}$, decreased by $0.5^{\circ} \mathrm{C}$ per day over 7 days. Feed and water were provided ad libitum.

\section{Data collection}

On BA $18 \mathrm{~d}$ and $20 \mathrm{~d}$ of LI, six eggs or chicks that hatched at peak hatching period (30\% to $70 \%$ hatch) were randomly sampled from each group for measurements of chick embryonic 
92

93

94

95

96

97

development. After eggs were broken open, the embryos or chicks were sacrificed by decapitation to obtain yolk weight and yolk free body weight (YFBW). Weights of heart, liver and stomach (gizzard and proventriculus) of all sampled embryos sacrificed on BA $18 \mathrm{~d}$ and $20 \mathrm{~d}$ of LI were determined.

The hatching time of individual eggs was determined using video recordings, and the hatching time was presented as biological age. From the first hatchling, number of chicks was recorded every an hour. The hatched chicks were removed from incubators every twelve hours to allow the camera to maintain a clear field of view. Hatch window was calculated by subtracting the hatching time of the last chick from that of the first chick. The peak hatching period was defined as $30 \%$ to $70 \%$ hatch of the batch. The total mortality was the number of dead embryos determined by candling on day 18 and unhatched eggs divided by the number of the fertile eggs.

At BA $504 \mathrm{~h}$ of LI (516 $\mathrm{h}$ of EI), 32 chicks per group which hatched in the peak hatching period were sampled and weighed. Metatarsus length (ML) was measured for assessment of leg bone development. After seven days' growth, all chickens received the same measurements to evaluate post-hatch development.

\section{Statistical analysis}

A one-way ANOVA model (SPSS 19.0) was used to analyze the effects of egg redistribution on the embryonic development of chicks (yolk free body weight, yolk weight, heart weight, liver weight and stomach weight) and post hatch performance (chick weight and tibia length). The level of significance was set at $\mathrm{P}<0.05$. The Fisher's LSD method was performed to test for overall differences among treatment groups. All data are shown as average \pm S. E. M. 
113

114

115

116

117

118

119

120

121

122

123

124

125

126

127

128

129

130

131

132

133

\section{RESULTS}

\section{Hatch performance}

The distribution of hatching time was obtained by video recordings of the four treatment groups. The EI group was found to give the first hatchling as expected, and the hatch window was 38 hours (Figure 1. A). However, the hatching process of EMI group started 5 hours later than that of EI group, while it finished at the same time as EI group (Figure 1. B). The start-up time of egg incubation in LI and LMI groups were 12 hours later than those of EI and EMI. As a result, the first chicks of LI and LMI groups emerged from eggs 2 and 8 hours later than EI group, respectively. The hatch process of LI group lasted 30 hours (Figure 1. C), 8 hours shorter compared to EI group. Moreover, LMI group had a shortened hatch window of 21 hours with highest hatchability $(95.8 \%)$, even though it started hatching at $468 \mathrm{~h}$ (Figure 1. D) which was 6 hours later than LI. According to $30 \%$ and $70 \%$ hatch time in Figure 2, the peak hatching period of manipulated incubation groups (EMI: $472.3-478.8$ h; LMI: $475.0-480.4$ h) was delayed 1.9 to 2.7 hours compared to the control groups (EI: 470.4 -477.0; LI: 472.1 - 478.7). Furthermore, the duration of the peak hatching period in EMI was the shortest (5.4 hours) and it was consistent to the narrow hatch window $(21 \mathrm{~h})$.

\section{Embryonic development from day 18 until hatch}

Embryonic development of the four groups on BA $18 \mathrm{~d}$ of LI was shown in Table 1. Yolk free body weights was higher in early incubation groups (EI and EMI) than those of late incubation groups (LI and LMI), but the yolk weights of early incubated eggs (EI and EMI) was found significantly lower than those of late incubated eggs (LI and LMI). In addition, organ size (heart 
134 weight and liver weight) was larger in EI and EMI, mainly caused by higher YFBW. However, no 135 significant difference for stomach weight was found.

136 The chicks of four incubation groups hatched in the peak hatching period had similar YFBW 137 (Table 2). Due to the earlier peak hatching period of chicks, yolk absorption of EI was faster and these chicks had higher liver and stomach weight. The LMI chicks that had short holding time in the incubator hatched with significantly higher yolk weight, lower liver and stomach weight. However, heart development of all hatched chicks was similar.

Overall, there were no significant differences between EI and EMI or LI and LMI in YFBW, yolk absorption and organ size. No effects of egg redistribution were observed for embryonic development both on BA $18 \mathrm{~d}$ and $20 \mathrm{~d}$ of LI.

\section{Post-hatch performance until day 7}

The evaluation of post-hatch performance until day 7 is presented in Table 3. At peak hatching time of LMI (480 h), body weight of chicks in early incubation groups (EI and EMI) was lower due to weight loss during the holding period in hatchers, while the EI and EMI chicks had higher

ML. However, no significant difference was found between EI and EMI, as well as between LI

and LMI. Similar results occurred after seven days' growth. Although both body weight and ML of early incubation groups (EI and EMI) were slightly higher than those of late incubation groups (LI and LMI), post-hatch growth and leg bone development was not altered by the manipulation of egg redistribution.

\section{DISCUSSION}

The aim of this study was to investigate the effects of egg redistribution during incubation on 
155

156

157

158

159

160

161

162

163

164

165

166

167

168

169

170

171

172

173

174

175

hatching time and post-hatch development. The results demonstrate that mixing eggs of different

developmental stages during incubation influenced the hatching process, including delayed

hatching time and shortened hatch window. They also suggest that embryonic development and

post-hatch performance were not altered by the egg redistribution on BA $12 \mathrm{~d}$ of LI.

The hatching time is known to be influenced by factors such as parental age, egg storage time

and conditions, and incubation conditions (Careghi et al. 2005; Decuypere \& Bruggeman 2007;

Tona et al. 2003). The hatching time distribution also results in different chick qualities and physiological characteristics of one batch of hatched chicks (Careghi et al. 2005; Wang et al.

2014). To eliminate these factors, the eggs were obtained from a single breeder flock, laid on the

same day, stored with very short time (no more than 2 days), and incubated in incubators with temperature and relative humidity calibration. Thus, the manipulation of egg redistribution on day

12 was presumed to be the only factor that affects the hatching time in this study.

The present study confirmed that mixing eggs of different growth curves shortened the hatch

window of the redistributed group, which is consistent with hatching synchronization found in pheasants (Persson \& Andersson 1999). The onset of the hatching process of redistributed eggs was retarded 5 to 6 hours, indicating that the narrow hatch window was related to the delay of the first hatch in manipulated group. This might be explained by some kind of egg communication between early incubated eggs and late incubated eggs. Chick embryos begin to develop a functionary auditory system as early as on day 10 of incubation (Alladi et al. 2002). Specific interaction among the redistributed eggs may take place after mixing eggs, by means of embryo sound communication. Perception of vocalizations by embryos may lead to physiological or 
176 behavioral changes. This is consistent with the finding of Tong (2015) that internal piping time

177 was delayed when embryos were exposed to manmade sound stimulation of embryos. However,

178 increased mortality was observed in duck and chicken eggs that were incubated under artificial

179 sound stimulation (Tong et al. 2015a; Veterany et al. 1999). Compared to the artificial sound

180 stimulation, embryo vocalization may impose less stress on other hatching eggs and exerts no

181 negative impact on hatchability. Another hypothesis is that environmental $\mathrm{CO}_{2}$ alters the hatch

182 process and results in a narrow spread of hatch. Previous researchers reported that high levels of

$183 \mathrm{CO}_{2}$ during the early stages of incubation stimulated early hatching and shortened hatch window

(De Smit et al. 2006; Tona et al. 2007). Although the onset of hatching process of mixed eggs was

delayed compared to the control groups, this did not extend the spread of hatch. The early

incubated embryos may penetrate the membrane and eggshell, and generate more $\mathrm{CO}_{2}$ during the

hatching period, leading to increased $\mathrm{CO}_{2}$ concentration that stimulated the hatching process of

late incubated eggs. Furthermore, the increasing $\mathrm{CO}_{2}$ concentration potentially contributes to the

hatchability of LMI (95.8\%) - higher than the other groups - suggesting that more chick embryos

succeeded in breaking out of eggshell rather than died in this difficult process. Considering this

delayed onset of the hatching process, the narrow spread of hatch and the increased hatchability,

our future work will focus on identifying to what degree, and via which mechanisms, redistributing

eggs of different growth curves affects hatching pattern and hatchability.

The advanced embryonic development of early incubated eggs was observed in both control

(EI) and manipulated group (EMI), mainly caused by the initial incubation time difference of 12

hours. However, mixing eggs of different growth curves did not alter the embryonic growth and 
197 yolk absorption before hatch. Chick embryos of both early incubated and late incubated eggs were

198 able to maintain normal organ development and nutrient metabolism until hatch. Although the

199 earlier hatched chicks (EI and EMI) underwent a longer holding period in incubators, the decreased

200 yolk weight and increased organ weight indicated that they got advanced maturation of organs

201 after hatching, as supposed by previous studies (Pinchasov \& Noy 1993; Tong et al. 2015b; Van

202 de Ven et al. 2011). No access to feed and water (EI and EMI, 36 h; LI and LMI, 24 h) for a long

203 time resulted in a higher weight loss in early incubation groups (EI and EMI), but enhanced the

204 leg bone development. The consistency of body weight and leg bone development on day 7 was

205 observed as expected. Nevertheless, the narrow hatch window of manipulated groups did not

206 influence chick growth performance up to day 7, indicating that egg distribution only stimulates

207 the hatching behavior. However, there is no evidence that response to eggs or egg communication

208 by egg distribution was related to this shortened hatch window. As reported above, there was no

209 negative effect of mixing eggs of different growth curves on embryonic growth, utilization of

210 nutrients and post-hatch performance.

\section{CONCLUSION}

212 The specific manipulation of mixing eggs of different initial incubation time influenced the

213 hatching pattern of late incubated eggs, including advanced hatching process and narrow hatch

214 window, but did not affect normal embryonic development, utilization of nutrients and post-hatch

215 performance of the late incubated eggs. All of these results are applicable in the industrial hatchery

216 to shorten hatch window and improve uniformity of chicks.

\section{ACKNOWLEDGEMENTS}


Li (College of Quality \& Safety Engineering, China Jiliang University) for his suggestions.

220

221

222

223

224

225

226

227

228

229

230

231

232

233

234

235

236

237

238

239

240

241

242

243

244

245

246

247

248

249

250

251

252

253

254

\section{REFERENCES}

Alladi P, Wadhwa S, and Singh N. 2002. Effect of prenatal auditory enrichment on developmental expression of synaptophysin and syntaxin 1 in chick brainstem auditory nuclei. Neuroscience 114:577-590.

Careghi C, Tona K, Onagbesan O, Buyse J, Decuypere E, and Bruggeman V. 2005. The effects of the spread of hatch and interaction with delayed feed access after hatch on broiler performance until seven days of age. Poultry Science 84:1314-1320.

Davies JC, and Cooke F. 1983. Intraclutch hatch synchronization in the lesser snow goose. Canadian Journal of Zoology 61:1398-1401.

De Smit L, Bruggeman V, Tona JK, Debonne M, Onagbesan O, Arckens L, De Baerdemaeker J, and Decuypere E. 2006. Embryonic developmental plasticity of the chick: Increased CO 2 during early stages of incubation changes the developmental trajectories during prenatal and postnatal growth. Comparative Biochemistry and Physiology Part A: Molecular \& Integrative Physiology 145:166-175.

Decuypere E, and Bruggeman V.2007. The endocrine interface of environmental and egg factors affecting chick quality. Poultry Science 86:1037-1042.

Decuypere E, Tona K, Bruggeman V, and Bamelis F. 2001. The day-old chick: a crucial hinge between breeders and broilers. World's Poultry Science Journal 57:127-138.

Holmberg K. 1991. Mallard ducks-mate choice and breeding success [Anas platyrhynchos]: Stockholm Univ.

Ipek A, and Sozcu A. 2017. Comparison of hatching egg characteristics, embryo development, yolk absorption, hatch window, and hatchability of Pekin Duck eggs of different weights. Poultry Science 96:3593-3599.

Maatjens C, Reijrink I, Molenaar R, Van Der Pol C, Kemp B, and van den Brand H. 2014. Temperature and $\mathrm{CO} 2$ during the hatching phase. I. Effects on chick quality and organ development. Poultry Science 93:645-654.

Nangsuay A, Meijerhof R, Van den Anker I, Heetkamp M, Morita VDS, Kemp B, and Van Den Brand H. 2016. Effects of breeder age, broiler strain, and eggshell temperature on development and physiological status of embryos and hatchlings. Poultry Science 95:16661679.

Persson I, and Andersson G. 1999. Intraclutch hatch synchronization in pheasants and mallard ducks. Ethology 105:1087-1096.

Pinchasov Y, and Noy Y. 1993. Comparison of post - hatch holding time and subsequent early performance of broiler chicks and Turkey poults. British poultry science 34:111-120.

Tona K, Bamelis F, De Ketelaere B, Bruggeman V, Moraes V, Buyse J, Onagbesan O, and 
Decuypere E. 2003. Effects of egg storage time on spread of hatch, chick quality, and chick juvenile growth. Poultry Science 82:736-741.

Tona K, Everaert N, Willemsen H, Gbeassor M, Decuypere E, and Buyse J. 2013. Effects of interaction of incubator $\mathrm{CO} 2$ levels and mixing hatching eggs of different embryo growth trajectory on embryo physiological and hatching parameters. British poultry science 54:545-551.

Tona K, Onagbesan O, Bruggeman V, De Smit L, Figueiredo D, and Decuypere E. 2007. Non-ventilation during early incubation in combination with dexamethasone administration during late incubation: 1. Effects on physiological hormone levels, incubation duration and hatching events. Domestic animal endocrinology 33:32-46.

Tong Q, McGonnell I, Romanini C, Bergoug H, Roulston N, Exadaktylos V, Berckmans D, Bahr C, Guinebretière M, and Eterradossi N. 2015a. Effect of species-specific sound stimulation on the development and hatching of broiler chicks. British poultry science 56:143-148.

Tong Q, McGonnell I, Roulston N, Bergoug H, Romanini C, Garain P, Eterradossi N, Exadaktylos V, Bahr C, and Berckmans D. 2015b. Higher levels of CO2 during late incubation alter the hatch time of chicken embryos. British poultry science 56:503-509.

Van de Ven L, Van Wagenberg A, Debonne M, Decuypere E, Kemp B, and Van Den Brand H. 2011. Hatching system and time effects on broiler physiology and posthatch growth. Poultry Science 90:1267-1275.

Veterany L, Hluchý S, and Weis J. 1999. The influence of sound stimulation during hatching on the mortality of ducks. Acta Physiologica Hungarica 86:105-110.

Vince MA. 1964. Synchronization of hatching in American bobwhite quail (Colinus virginianus). Nature 203:1192-1193.

Vince MA. 1968. Retardation as a factor in the synchronization of hatching. Animal Behaviour 16:332-335.

Wang Y, Li Y, Willems E, Willemsen H, Franssens L, Koppenol A, Guo X, Tona K, Decuypere E, and Buyse J. 2014. Spread of hatch and delayed feed access affect post hatch performance of female broiler chicks up to day 5. Animal 8:610-617.

Willemsen H, Debonne M, SWENNEN Q, Everaert N, Careghi C, Han H, Bruggeman V, Tona K, and Decuypere E. 2010a. Delay in feed access and spread of hatch: importance of early nutrition. World's Poultry Science Journal 66:177-188.

Willemsen H, Kamers B, Dahlke F, Han H, Song Z, Ansari Pirsaraei Z, Tona K, Decuypere E, and Everaert N. 2010b. High-and low-temperature manipulation during late incubation: Effects on embryonic development, the hatching process, and metabolism in broilers. Poultry Science 89:2678-2690. 
Figure 1

Hatching pattern of chicks in four groups, including distribution of hatching time (BA), hatch window and hatchability

(A) Early incubation group(EI) ; (B) Early incubated eggs in manipulated group (EMI); (C) Late incubation group (LI) ; (D) Late incubated eggs in manipulated group (LMI).
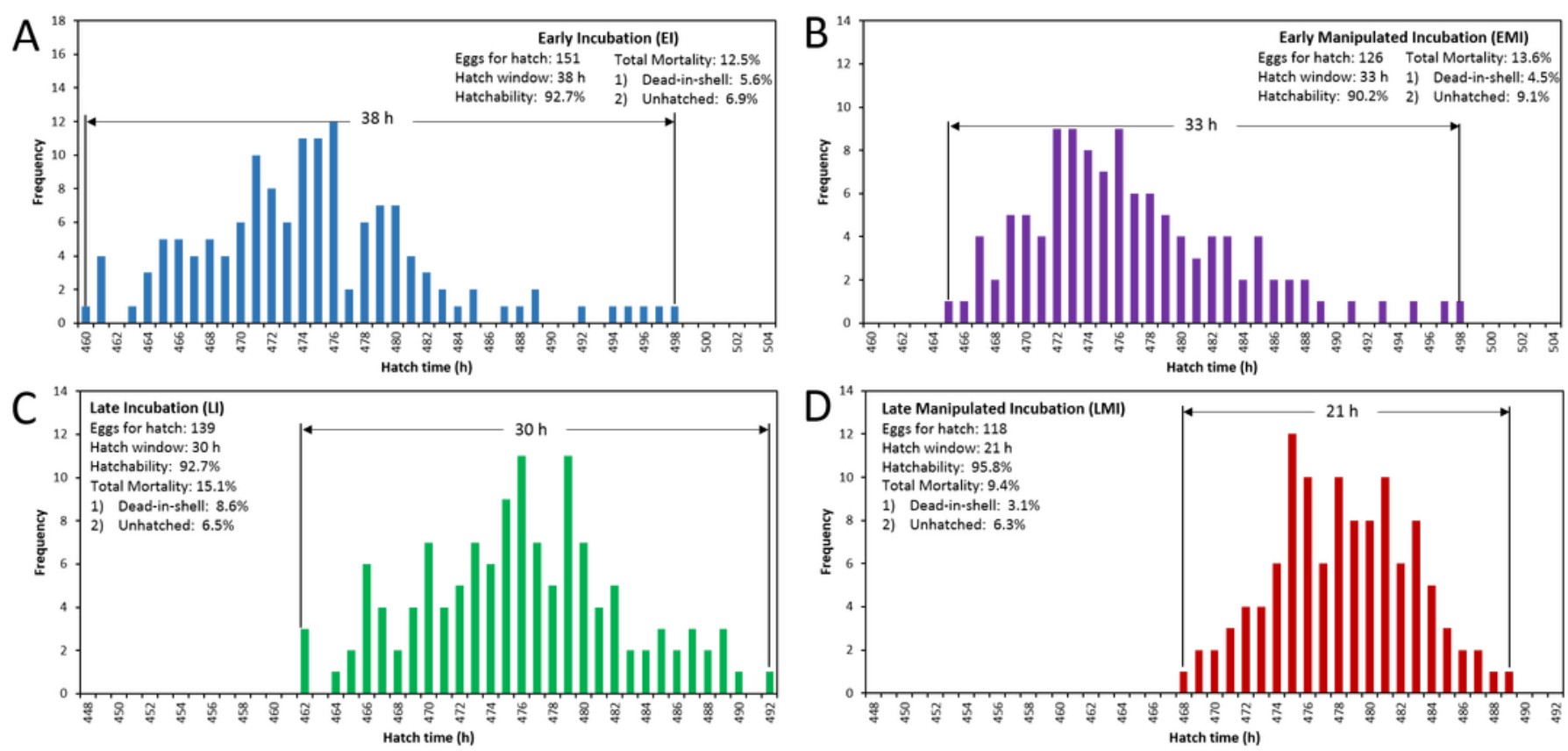
Figure 2

Hatch accumulation of four groups and peak hatching period which defined as $30 \%$ to $70 \%$ hatch.

The red line (EMI) indicates the latest onset of hatching process with shortest peak hatching period.

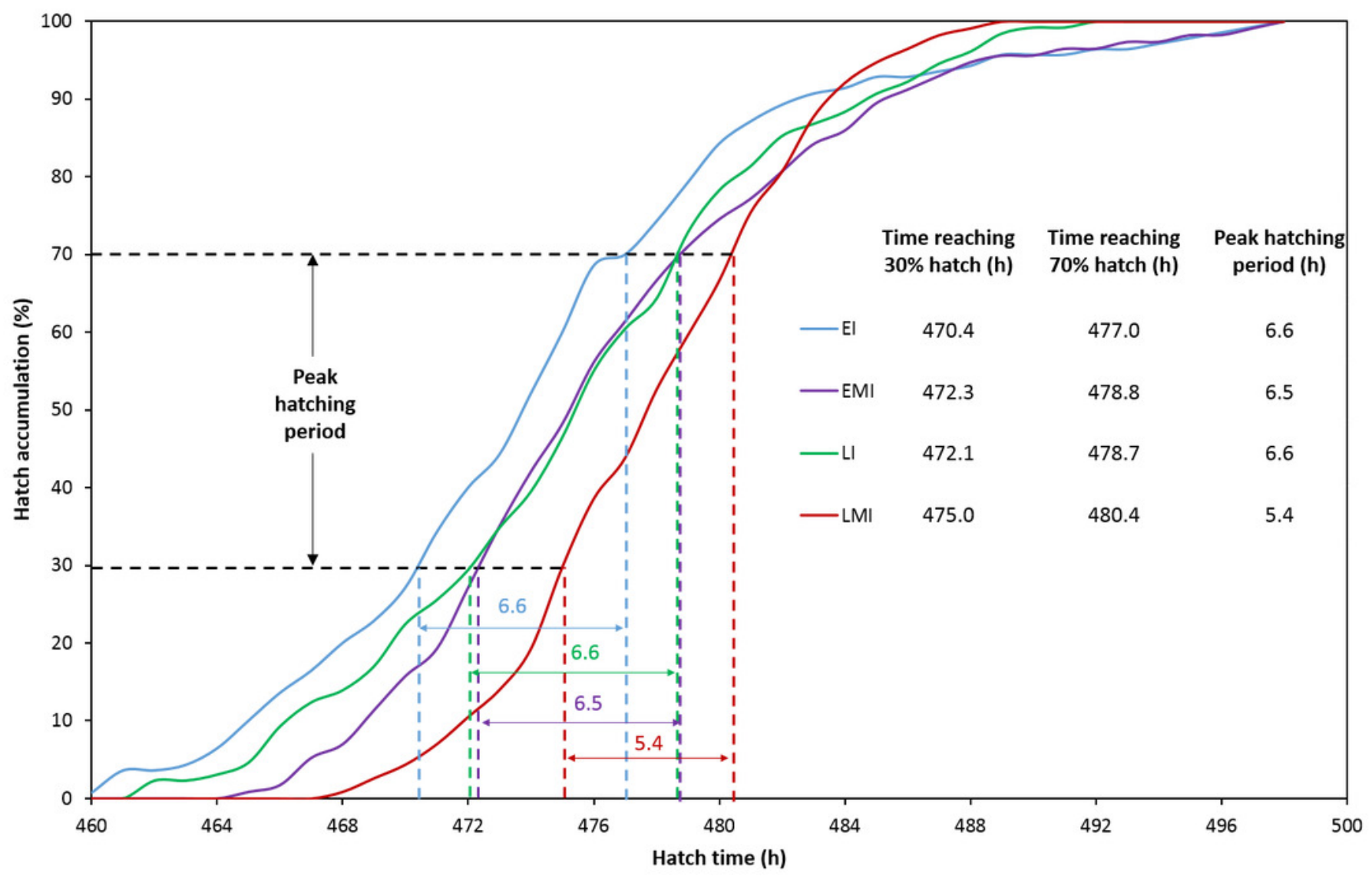




\section{Table $\mathbf{1}$ (on next page)}

Embryonic development, yolk absorption and organ weight of chick embryos on BA $18 \mathrm{~d}$ of LI. 
Table 1. Embryonic development, yolk absorption and organ weight of chick embryos on BA

$18 \mathrm{~d}$ of LI.

\begin{tabular}{cccccc}
\hline & \multicolumn{2}{c}{ Early incubated eggs } & \multicolumn{2}{c}{ Late incubated eggs } & \multirow{2}{*}{ P value } \\
\cline { 2 - 5 } & Control & Manipulated & Control & Manipulated & \\
\hline YFBW $(\mathrm{g})$ & $30.27 \pm 0.63 \mathrm{a}$ & $30.50 \pm 0.57 \mathrm{a}$ & $27.68 \pm 0.74$ & $28.41 \pm 0.79$ & $<0.05$ \\
& & & $\mathrm{~b}$ & $\mathrm{~b}$ & \\
Yolk weight $(\mathrm{g})$ & $15.16 \pm 0.59 \mathrm{~b}$ & $15.46 \pm 0.51$ & $16.31 \pm 0.45 \mathrm{a}$ & $16.69 \pm 0.33 \mathrm{a}$ & $<0.05$ \\
& & $\mathrm{~b}$ & & & \\
Heart weight $(\mathrm{g})$ & $0.16 \pm 0.01 \mathrm{a}$ & $0.16 \pm 0.01 \mathrm{a}$ & $0.14 \pm 0.01 \mathrm{~b}$ & $0.15 \pm 0.01 \mathrm{~b}$ & $<0.05$ \\
Liver weight $(\mathrm{g})$ & $0.47 \pm 0.02 \mathrm{ab}$ & $0.51 \pm 0.02 \mathrm{a}$ & $0.44 \pm 0.04 \mathrm{bc}$ & $0.42 \pm 0.01 \mathrm{c}$ & $<0.05$ \\
$\begin{array}{c}\text { Stomach weight } \\
\text { (g) }\end{array}$ & $0.95 \pm 0.06$ & $0.95 \pm 0.15$ & $0.92 \pm 0.03$ & $0.91 \pm 0.11$ & $>0.05$ \\
\hline
\end{tabular}




\section{Table 2 (on next page)}

Embryonic development, yolk absorption and organ weight of hatched chicks on BA 20 $\mathrm{d}$ of LI. 
Table 2. Embryonic development, yolk absorption and organ weight of hatched chicks on BA $20 \mathrm{~d}$ of LI.

\begin{tabular}{cccccc}
\hline & \multicolumn{2}{c}{ Early incubated eggs } & \multicolumn{2}{c}{ Late incubated eggs } & \multirow{2}{*}{ P value } \\
\cline { 2 - 5 } & Control & Manipulated & Control & Manipulated & \\
\hline YFBW $(\mathrm{g})$ & $31.99 \pm 0.89$ & $31.38 \pm 0.92$ & $31.50 \pm 0.65$ & $31.94 \pm 0.53$ & $>0.05$ \\
Yolk weight $(\mathrm{g})$ & $4.04 \pm 0.26 \mathrm{c}$ & $4.65 \pm 0.20 \mathrm{ab}$ & $4.45 \pm 0.23 \mathrm{~b}$ & $4.89 \pm 0.10 \mathrm{a}$ & $<0.05$ \\
Heart weight $(\mathrm{g})$ & $0.27 \pm 0.01$ & $0.27 \pm 0.01$ & $0.27 \pm 0.01$ & $0.26 \pm 0.01$ & $>0.05$ \\
Liver weight $(\mathrm{g})$ & $0.93 \pm 0.02 \mathrm{a}$ & $0.88 \pm 0.04 \mathrm{ab}$ & $0.85 \pm 0.04 \mathrm{~b}$ & $0.79 \pm 0.02 \mathrm{c}$ & $<0.05$ \\
Stomach weight & $4.00 \pm 0.05 \mathrm{a}$ & $3.86 \pm 0.26 \mathrm{ab}$ & $3.81 \pm 0.13 \mathrm{~b}$ & $3.35 \pm 0.11 \mathrm{c}$ & $<0.05$ \\
$(\mathrm{~g})$ & & & & & \\
\hline
\end{tabular}




\section{Table 3 (on next page)}

Post-hatch body growth and leg bone development up to day 7 . 
Table 3. Post-hatch body growth and leg bone development up to day 7.

\begin{tabular}{cccccc}
\hline & \multicolumn{2}{c}{ Early incubated eggs } & \multicolumn{2}{c}{ Late incubated eggs } & P value \\
& Control & Manipulated & Control & Manipulated & \\
\hline Day 0 & & & & & \\
Body weight (g) & $36.80 \pm 0.77 \mathrm{~b}$ & $37.24 \pm 0.65 \mathrm{~b}$ & $38.43 \pm 0.77 \mathrm{a}$ & $38.73 \pm 0.61 \mathrm{a}$ & $<0.05$ \\
ML (mm) & $21.64 \pm 0.44 \mathrm{a}$ & $21.53 \pm 0.35 \mathrm{a}$ & $20.85 \pm 0.40 \mathrm{~b}$ & $20.82 \pm 0.34 \mathrm{~b}$ & $<0.05$ \\
$\quad$ Day 7 & & & & & \\
Body weight (g) & $72.66 \pm 1.89$ & $72.74 \pm 1.76$ & $72.13 \pm 1.61$ & $71.83 \pm 1.25$ & $>0.05$ \\
ML (mm) & $31.39 \pm 0.45 \mathrm{a}$ & $31.70 \pm 0.57 \mathrm{a}$ & $30.93 \pm 0.57 \mathrm{~b}$ & $30.78 \pm 0.63 \mathrm{~b}$ & $<0.05$ \\
\hline
\end{tabular}

